\title{
DISTRIBUTION OF BLUE GALAXIES IN A MERGING CLUSTER OF GALAXIES ABELL $168^{1}$
}

\author{
AKIHIKO TOMITA, FUMITAKA E. NAKAMURA, TADAFUMI TAKATA², \\ KOUICHIROU NAKANISHI, TSUTOMU TAKEUCHI, AND KOUJI OHTA \\ Department of Astronomy, Faculty of Science, Kyoto University, \\ Sakyo-ku, Kyoto 606-01, Japan \\ Electronic mail: atomita, fuming, takata, nakanisi, takeuchi, ohta@kusastro.kyoto-u.ac.jp \\ and \\ TORU YAMADA \\ Cosmic Radiation Laboratory, The Institute of Physical and Chemical Research (RIKEN), \\ Wako, Saitama 351-01, Japan \\ Electronic mail: yamada@cricket.riken.go.jp
}

Received 10 April 1995; accepted

Submitted to the Astronomical Journal

\footnotetext{
${ }^{1}$ Based on observations made at Kiso Observatory. Kiso Observatory is operated by Institute of Astronomy, Faculty of Science, University of Tokyo, Japan.

${ }^{2}$ Present address: National Astronomical Observatory, Mitaka, Tokyo 181, Japan; Electronic mail: takata@optik.mtk.nao.ac.jp
} 


\begin{abstract}
The fraction of star-forming galaxies in rich clusters of galaxies increases rapidly with the redshift. This is interpreted as the result of a rapid evolution of cluster galaxies, though its mechanism is not yet clear. One hypothesis is that if galaxies run into the dense intracluster medium (ICM) regions, starbursts can be induced due to compression of molecular clouds in the galaxies by a raised "external" ICM pressure. In a merging cluster, there would be dense ICM regions and some galaxies may experience a rapid increase of external pressure. Thus, if the mechanism works, starbursts would occur in such galaxies. Such a situation is considered to be realized in Coma cluster, and galaxies showing a recent starburst have a characteristic spatial distribution; they populate an elongated region between two sub-clusters. We examined the above hypothesis by measuring the spatial distribution of blue galaxies, regarded as star-forming galaxies in Abell 168 to check whether a case like Coma is realized in this recently merging cluster. However, we could not find an enhanced blue-galaxy distribution between two sub-clusters. We discuss the causes of our result, including possibilities of surviving the hypothesis.
\end{abstract}




\section{Introduction}

Butcher \& Oemler $(1978,1984)$ claimed that the fraction of blue galaxies in rich clusters of galaxies rapidly increases to about one fourth at a redshift of 0.5 from only a few percent at present; this is the so-called Butcher-Oemler effect. Subsequent spectroscopic observations (Dressler \& Gunn 1982, 1983, 1992; Dressler et al. 1985; Ellis et al. 1985; Lavery \& Henry 1986; Henry \& Lavery 1987; Couch \& Sharples 1987; MacLaren et al. 1988; Mellier et al. 1988; Fabricant et al. 1991) have revealed that most of these blue galaxies are related to strong star-forming activity (for a review, see Dressler \& Gunn 1988). In the present-day rich clusters, almost all cluster members are gas-poor E/S0 galaxies and do not show star-forming activities. Thus, there may have been a rapid evolution of cluster galaxies. However, the mechanism responsible for this rapid evolution is not yet clear.

Dressler \& Gunn (1983) suggested a possibility that this rapid evolution is caused by the interaction with the intracluster medium (ICM); gas-rich galaxies fall into the central region of a cluster for the first time and they experience a rapid increase of ICM "external" pressure, quickly exceeding the ISM "internal" pressure, which triggers the starburst by compressing the molecular clouds in galaxies (first infall model; see also Bothun \& Dressler 1986; Dressler \& Gunn 1988; Gunn 1989). Evrard (1991) showed from his simulations that the first infall model can explain the Butcher-Oemler effect semi-quantitatively. However, it is also possible that another mechanism, galaxy-galaxy interactions, may play an important role in causing the Butcher-Oemler effect (Lavery \& Henry 1988, 1994; Lavery et al. 1992). It is necessary to examine whether the mechanism, running into high pressure-ICM region, can actually induce the starbursts in cluster members.

Caldwell et al. (1993) found from their spectroscopic observations that in the Coma cluster strong Balmer-line-absorption galaxies reside in the elongated region between two x-ray peaks, the main Coma cluster and the NGC 4839 sub-cluster (see their Fig. 17). They 
pointed out that the strong Balmer-line-absorption galaxies are similar to post-starburst galaxies observed in the "Butcher-Oemler" clusters. Burns et al. (1994) showed from their simulations that the sub-cluster of Coma, which is now seen as the second x-ray peak, penetrated the main Coma cluster 2 Gyr ago. They also calculated that the penetrating sub-cluster members would spread from the center of the main cluster in the direction of the sub-cluster, and that their distribution is similar to that of the Balmer-line-absorption galaxies found by Caldwell et al. (1993). This suggests that the sub-cluster members have traveled through the main cluster, and suffered a higher pressure of the ICM inducing a burst of star formation in these galaxies. This phenomenon can be understood in the same way as the first infall model; this time passing through the main cluster gives the same effect to galaxies as infalling toward the cluster center. Merging clusters must be a suitable laboratory for testing the first infall model.

We examined a distribution of blue galaxies, regarded as star-forming galaxies, in a merging cluster of galaxies, Abell 168. If gas-rich galaxies have orbits on which a sudden change of the ICM pressure can occur, inducing starbursts, we would expect to see a second Coma case, i.e., the blue galaxies would spread in the region from the main cluster center to the direction of the sub-cluster. The paper is organized as follows: the characteristics of the target cluster, Abell 168, are given in Sec. 2. Observations and data reduction including the discussion on the completeness of our sample are given in Sec. 3. In Sec. 4, we describe our catalog of observed galaxies. Distribution and morphology of the "blue" galaxies are described in Sec. 5 and 6, respectively. Some comments on dominant galaxies are given in Sec. 7. Discussion follows in Sec. 8, and we summarize briefly in Sec. 9.

\section{Target Cluster Abell 168}


Abell 168 has a redshift of 0.04479 and this corresponds to $131 \mathrm{Mpc}$ (we use $H_{0}=100 \mathrm{~km} \mathrm{~s}^{-1} \mathrm{Mpc}^{-1}$ and $q_{0}=0$ throughout this paper). This is very feasible distance for covering a large area of the cluster by several CCD fields and resolving the individual galaxies to be distinguished them from stars. The field of view of our CCD chip is about $13^{\prime}$ square, while the order of magnitude of the cluster size, $1 \mathrm{Mpc}$, corresponds to $27^{\prime}$. And a seeing size is about $4^{\prime \prime}$ (see Sec. 3) which is fearly smaller than the typical size of small galaxies, $5 \mathrm{kpc} \sim 8^{\prime \prime}$. Our limiting maganitude is $19 \mathrm{mag}(\operatorname{See} \mathrm{Sec} .3)$. The distance modulus $(m-M)$ of 35.7 leads to absolute magnitude of -16.7 mag which is fainter than that of SMC. The characteristics of Abell 168 are summarized in Table 1.

Abell 168 is an example of clusters in the process of merging. Ulmer et al. (1992) have investigated offsets between x-ray and optical (galaxy distribution) centers of rich clusters of galaxies. Out of 13 clusters they studied, Abell 168 was found to be the most extreme case; x-ray/optical offset is $400 \mathrm{kpc}$ (see their Fig. 2). They suggested that Abell 168 was formed by the collision of two approximately equal-sized clusters with different gas-to-galaxy ratios. Background and foreground galaxy contamination was examined by Zabludoff et al. (1993) by measuring redshifts of galaxies in the direction of Abell 168. Although they found a foreground group in the southern region of the cluster, there is no strong contamination in the central region. Thus, it is reliable that the x-ray/optical discrepancy is real and x-ray multipeaks exist in Abell 168.

The hot gas is thought to be governed by the gravitational potential of the dark matter and the typical time scale within which the multipeaks would vanish is comparable to the sound crossing time. Around x-ray peaks, the temperature of the hot gas is about $3 \times 10^{7}$ K (David et al. 1993) and the distance between peaks is about $400 \mathrm{kpc}$. The calculated sound crossing time around this region is about 0.6 Gyr. Therefore, not more than 0.6 Gyr could have passed after the collision in order to see the complex x-ray distribution and 
the x-ray/galaxy discrepancy; Abell 168 is dynamically younger than Coma. The number of member galaxies within a magnitude range $m_{3}$ to $m_{3}+2$ is 89 for Abell 168 and 106 for Coma, where $m_{3}$ is the magnitude of the third brightest galaxy in each cluster. This means that Abell 168 is nearly as rich as Coma. On the other hand, x-ray temperature of the ICM is $2.6 \mathrm{keV}$ for Abell 168 (David et al. 1993) and this is quite lower than that for Coma, $8.3 \mathrm{keV}$. Assuming that the mass-to-luminosity ratio of two clusters does not so much differed from each other, this suggests that Abell 168 is not yet virialized. This also indicates that Abell 168 is a dynamically young system.

\section{Observations and Data Reduction}

We made $V$ and $I$ band CCD photometry of the central $0.18 \mathrm{deg}^{2}$ of Abell 168 in November and December 1993 using the 1.05-m Kiso Schmidt telescope (F/3.1) equipped with a single-chip CCD camera in the prime focus. The CCD chip has $1000 \times 1018$ pixels and one pixel size corresponds to $0 . " 752$, giving a field-of-view of $12 .^{\prime} 5 \times 12 .^{\prime} 7$. The total observed region was covered by seven individual CCD fields; the central position and a total exposure time as well as a seeing size, FWHM of stellar images in the reduced frame of each field (about three to four arcsec), are tabulated in Table 2. Our search field is shown in Fig. 1 superimposed on the x-ray/galaxy map of Fig. 2 in Ulmer et al. (1992). The Kiso-CCD system has a good sensitivity at $V, R$, and $I$ bands. The expected color change during the period of $10^{9} \mathrm{yr}$ after the starburst is about twice as large in $(V-I)$ color than in $(V-R)$ color (see e.g., Arimoto \& Yoshii 1986). Therefore, we chose to observe in the $V$ and $I$ bands; filters for Johnson $V$ and Kron-Cousins $I$ band were used.

For data reduction and analysis, we used IRAF 3 and SPIRAL (Hamabe \& Ichikawa

\footnotetext{
${ }^{3}$ IRAF is the software developed in National Optical Astronomy Observatories.
} 
$1992)$ in the usual manner. After bias-subtraction and flat-fielding with dome-flat frames using IRAF, sky-subtraction was made with SPIRAL. Several object frames were combined to remove radiation events and to improve the signal-to-noise ratio. Object detection was made by IRAF tasks in DAOPHOT packages. Typically about 150 objects were detected in each CCD field. The faintest ones had magnitudes of about 20.5 and 19.5 in $V$ and $I$ bands, respectively. We will describe the details of photometry, discrimination between galaxies and stars, and completeness of the sample below.

By using photometric standard star (Feige 11) frames (Landolt 1992), we converted CCD count rates into magnitude ( $V$ : Johnson, $I$ : Kron-Cousins). We made photometry by following the equations:

$$
\begin{aligned}
& V_{\text {Landolt }}=v+c_{\mathrm{V} 1}+c_{\mathrm{V} 2} F(z)+c_{\mathrm{V} 3}(V-I), \\
& I_{\text {Landolt }}=i+c_{\mathrm{I} 1}+c_{\mathrm{I} 2} F(z)+c_{\mathrm{I} 3}(V-I),
\end{aligned}
$$

where $v$ and $i$ indicate $-2.5 \log$ (count rate) on CCD, $F(z)$ is the air mass function. The coefficients $c_{\mathrm{V} 1}, c_{\mathrm{I} 1}, c_{\mathrm{V} 2}$, and $c_{\mathrm{I} 2}$ were determined for each night. We used $c_{\mathrm{V} 3}=0.07$ and $c_{\mathrm{I} 3}=-0.03$ for all observation nights; these values were measured by observing five stars with different colors in the PG 0942-029 field (Landolt 1992). We performed aperture photometry using the IRAF tasks in APPHOT package; the aperture radius adopted was 10 arcsec (about 3 times point source FWHM, see Table 2) for both $V$ and $I$ band images. In the fourth column of Table 3 (the catalog which will be explained in Sec. 4), we list the size of the galaxies defined as the major axis at $23 \mathrm{mag} \operatorname{arcsec}^{-2}$ isophote in $I$ band. If it exceeds 20 arcsec, we took the aperture radius of one half of the major-axis size. We estimated the error of the photometry by comparing the data for a given object taken in more than two frames; the root-mean-square of the errors in 1 mag bin are shown in Fig. 2 (a) for $V$ band, and Fig. 2 (b) for $I$ band. A typical error of photometry is $\Delta V \sim 0.05$ mag and $\Delta(V-I) \sim 0.08 \mathrm{mag}$ at $V=18 \mathrm{mag}$. Sky brightness was about $20.8-21.3$ and $19.2-$ 
19.7 mag $\operatorname{arcsec}^{-2}$ in $V$ and $I$ band, respectively.

In order to distinguish galaxy images from stellar images, we constructed two diagrams; Diagram A (Fig. 3) and Diagram B (Fig. 4). Diagram A shows peak count versus FWHM of the images and Diagram B shows peak/FWHM² versus magnitude. Open and filled circles in both diagrams indicate what we could identify by eye inspection as galaxies or foreground stars, respectively. Crosses indicate cases, where we could not reliably assign any category. In both diagrams, a galaxy sequence and a stellar sequence clearly separate from each other and both sequences agree with the grouping by eye inspection fairly well. Therefore, we could distinguish galaxies from stars on CCD images down to about 19 mag in $V$ band and about $18 \mathrm{mag}$ in $I$ band. Finally, we detected 143 galaxies brighter than $V \sim 19$ mag.

The galaxy luminosity function in Abell 168 was studied by Dressler (1978) ( $F$ band) and Oegerle et al. (1986) ( $r$ band). Fig. 5 shows the differential luminosity function in the $V$ band of our sample including background contamination (filled circles) and that in the central $0.2 \mathrm{deg}^{2}$ by Dressler (1978) (open squares) and by Oegerle et al. (1986) (open triangles). Dressler (1978) and Oegerle et al. (1986) subtracted the backgroundcontamination by using the field galaxy number density data in the $J$ band by Oemler (1974); this background data reaches down to about 20 mag. A solid line in Fig. 5 indicates the general background count by Oemler (1974). We converted the magnitude in different color bands assuming that $(J-V)=0.3 \mathrm{mag},(V-F)=0.7 \mathrm{mag}$, and $(V-r)=0.27 \mathrm{mag}$ (Oemler 1974; Oegerle et al. 1986). Our luminosity function reproduces that by Dressler (1978) and Oegerle et al. (1986) in the bright part ( $\lesssim 18 \mathrm{mag})$ and follows the background count by Oemler $(1974)$ in the faint end $(\sim 19 \mathrm{mag})$. Thus, our galaxy count in the central Abell 168 region is consistent with these previous papers; our sample is found to be nearly complete down to about $V \sim 19$ mag. 
Finally we show that the depth of our search is suitable. The Butcher \& Oemler (1984) sample has a magnitude range of about five in each cluster, and there are many blue galaxies within this magnitude range. Our sample also covers the same magnitude range; the brightest magnitude in our sample is about $V=14$ mag and the faintest one is about $V=19$ mag. Strong Balmer-line-absorption galaxies in Coma cluster are found in a wide magnitude range and the faintest one among them has a magnitude of $V=17.2$ mag, which corresponds to $V=18.7 \mathrm{mag}$ at the distance of Abell 168. Therefore, we searched galaxies deeply enough to catch blue galaxies.

\section{The Catalog}

The catalog of 143 objects is given in Table 3. An ID-number is tabulated in the first column of Table 3. Position in 1950.0 equinox is given in the second and third columns; the position error is about 5 arcsec. The positions of the galaxies are determined from those of some bright galaxies previously known; the data are taken from NASA/IPAC Extragalactic Database (NED). The fourth column indicates the major axis size of a galaxy in $\operatorname{arcsec}$ at $23 \mathrm{mag} \operatorname{arcsec}^{-2}$ isophote in $I$ band; 1 arcsec corresponds to 610 pc. The $V$ and $I$ magnitudes and the $(V-I)$ color are given in the fifth, sixth, and seventh column, respectively. Eye-inspected morphology is assigned for each galaxy and is tabulated in the eighth column. Since most of the galaxies have sizes smaller than four times of the point source FWHM, we can not see the morphology in detail. Therefore, we only assign for morphology either of E, S0, early $\mathrm{S}(\mathrm{Se})$, late $\mathrm{S}(\mathrm{S} l)$, I; if we can see any bar structure, we indicate this by $\mathrm{SB} e$ or $\mathrm{SB} l$. If we could not assign unambiguously one of the categories, we write for instance, S0/Se. "?" is added if the classification is uncertain. We tabulate the distribution of morphologies in Table 4. Here we combine barred and non-barred, and uncertain assignments, like for instance $\mathrm{S} e$ ?, are also included into one category. Objects 
assigned to two categories are divided by number into each category; for instance, there are five E/S0 galaxies and we count here that there are 5/2 E's and 5/2 S0's. From Table 4, we find that there are many spiral galaxies; nearly two thirds are spirals and Abell 168 is a spiral-rich cluster. In the ninth column, we list the ID-number given in Dressler (1980). Radial velocities of 22 galaxies are measured by Faber \& Dressler (1977), Chapmann et al. (1988), and Zabludoff et al. (1993). We list heliocentric radial velocities in the tenth column; if more than two observations exist for one galaxy, we take the later one. Some comments are given in the last column. We use many abbreviations here; see caption.

\section{Distribution of the Blue Galaxies}

Figure 6 shows the $(V-I)$ color - $V$ magnitude diagram (C-M diagram). Most galaxies have a color of 1.2 to 1.4, and we can see a small color-magnitude (C-M) effect (i.e., bluer colors for fainter galaxies) on this diagram. Since we will examine the color distribution within a magnitude range of over five, the C-M effect is not negligible in defining the blue objects. We measured the C-M effect by fitting the points on Fig. 6 having $V=13.5$ $17.5 \mathrm{mag}$ and $(V-I)=1.1-1.5 \mathrm{mag}$ and obtained $\Delta(V-I) / \Delta V=0.032$.

Figure 7 presents the color distribution of Abell 168 galaxies after correcting the C-M effect; we converted observed Johnson-Kron-Cousins color into C-M effect-corrected color as follows:

$$
(V-I)_{\mathrm{corr}}=(V-I)+0.032\left(V-V_{\mathrm{C}}\right)
$$

where $V_{\mathrm{C}}$ is an arbitrary value, and here we adopt $17.0 \mathrm{mag}$ as a mid point of observed $V$ magnitudes; peak color is set to be $(V-I)_{\text {corr }}=1.2$ mag. Since the error of color is about $0.1 \mathrm{mag}$ in the worst case, the step of color in Fig. 7 is taken to be $0.1 \mathrm{mag}$. We can see in Fig. 7 a high concentration in $(V-I)_{\text {corr }}=1.1$ to 1.3 mag, which corresponds to the typical 
colors of early-type galaxies. Objects whose colors are $(V-I)_{\text {corr }}=1.0$ to 1.4 mag occupy about $80 \%$ of the total objects. There is also a small blue wing in Fig. 7 . We define as a color criterion for the blue objects that they are more than 0.2 mag bluer than the mode of the color distribution of $1.2 \mathrm{mag}$, i.e., bluer than $(V-I)_{\text {corr }}=1.0$ mag. Galaxies having blue colors of $1.0 \mathrm{mag}<(V-I)_{\text {corr }} \leq 1.1 \mathrm{mag}$ are denoted as semi-blue. In Fig. 6 the filled and shaded circles indicate the blue and semi-blue objects, respectively, and a solid line shows the typical color of early-type galaxies, $(V-I)_{\text {corr }}=1.2 \mathrm{mag}$. In Fig. 7 the filled and shaded areas show the regions for the blue and the semi-blue objects, respectively. Bica et al. (1990) calculated synthetic colors of galaxies with a partial (0.1, 1, and $10 \%$ mass) starburst superimposed on old, already existing populations. From their model of a ten percent mass-starburst, $\Delta(V-I)=0.2 \mathrm{mag}$ separates galaxies younger than $10^{9} \mathrm{yr}$ after the starburst from "old-color" galaxies f. Balmer-line-absorption galaxies in Coma cluster generally have blue $(B-V)$ colors; about 0.1 mag bluer in $(B-V)$ than the color mode after correcting the C-M effect. $\Delta(B-V) \sim 0.1$ mag corresponds to $\Delta(V-I) \sim 0.1 \mathrm{mag}$ in the 10 percent-mass partial-starburst model by Bica et al. (1990).

Figure 8 shows the distribution of galaxies in Abell 168 detected in this study superimposed on the x-ray (0.15-4.0 keV) intensity contours from Ulmer et al. (1992). The blue and semi-blue objects are indicated by the filled and shaded circles with their ID-numbers, respectively. The size of the circles is proportional to the size of the major axis of the galaxies, as tabulated in the fourth column of Table 3, though the scale is expanded twice. Although redshift measurements by Zabludoff et al. (1993) showed that there is no foreground contamination, there remains a problem of some background contamination.

\footnotetext{
4 Bica et al. (1990) gave the tables in Johnson system. In converting Johnson $(V-I)$ color to Johnson-Kron-Cousins $(V-I)$ color, we used the relation $(V-I)_{\mathrm{JKC}}=$ $(1 / 1.30)(V-I)_{\mathrm{J}}+0.01$ (Cousins 1976).
} 
Since we are searching for the blue object concentration in the cluster field, a uniform contamination does not affect our study, though, the contrast of the blue galaxy distribution may become smaller. We also list in Table 4 the blue fractions for each morphological type. They are 20 and $46 \%$ for early spirals and late spirals, respectively. Assuming that field galaxies equally consist of early and late spirals, and that the blue fraction in each morphological category for the field galaxies is the same as that for Abell 168 members, the blue fraction for the field galaxies would be $(20+46) / 2=33 \%$, almost one third. From Fig. 5 our sample is expected to have about 30 contamination galaxies with $V$ magnitude fainter than about 18.5 mag. So we have about ten blue contamination galaxies in our sample. It contains 23 blue galaxies, of which 14 galaxies have magnitudes brighter than 18.5 mag and 9 galaxies are fainter than that. Thus, all blue galaxies fainter than 18.5 mag may be background-contamination galaxies. The numbers with circles in Fig. 8 indicate the objects brighter than or equal to 18.5 mag; these galaxies are very likely cluster members.

From Fig. 8 we can see that the blue and semi-blue objects spread over the cluster and neither avoid the region between the two x-ray peaks nor concentrate in it. Though the number density of galaxies near the $\mathrm{cD}$ galaxy (No. 46) is not large, it seems that the blue galaxies do not tend to distribute around the $\mathrm{cD}$ galaxy. We show in Fig. 9 the blue and semi-blue galaxy fraction as a function of distance to the cluster center in units of the Abell radius $\left(R_{\mathrm{A}}[\operatorname{arcmin}] \equiv 1.7 z^{-1}\right)$. We assume that the cluster center is located at the maximum of the galaxy surface number density taken from data by Geller \& Beers (1982) (this point is indicated by a cross in Fig. 8); note that it is also nearly the center of our observed region. Filled and shaded areas are for the blue and semi-blue objects, respectively. Fig. 9 (a) contains all galaxies and Fig. 9 (b) only contains the brighter $(\leq$ $18.5 \mathrm{mag}$ ) galaxies. It seems that the blue galaxies tend to avoid the cluster center (within $0.1 R_{\mathrm{A}}$ ) but lie within $0.4 R_{\mathrm{A}}$, though the statistical error in the outer region is large. 
We call the first and second x-ray peaks Point A and Point B, respectively, as shown in Fig. 8. We assume that these two peaks represent the centers of sub-clusters. Though Point $\mathrm{B}$ is the second $\mathrm{x}$-ray peak, it is close to the location of the maximum of the galaxy surface number density. The central x-ray surface brightnesses of Point A and Point B are $9 \times 10^{-14}$ and $3 \times 10^{-14} \mathrm{erg} \mathrm{s}^{-1} \mathrm{~cm}^{-2} \operatorname{arcmin}^{-2}(0.15-4.0 \mathrm{keV})$, respectively (tabulated in Table 1). Note that for Coma, the peak x-ray surface brightness is higher, $2.3 \times 10^{-13}$ $\mathrm{erg} \mathrm{s}^{-1} \mathrm{~cm}^{-2} \operatorname{arcmin}^{-2}(0.15-4.0 \mathrm{keV})$; this reflects that the virialization is more developed in Coma, as mentioned in Sec. 2. For Coma, the central x-ray surface brightness ratio of the main to the sub-cluster is ten, while for Abell 168, the ratio is three. Therefore, unlike the Coma case, in Abell 168 the richness of the northern and southern sub-clusters are not so different from each other. A reference direction is defined by a line connecting the centers of both sub-clusters. Centered on either Point A or Point B, we measure angles counter-clockwise from the reference direction mentioned above. We performed an angular variation analysis here with respect to both Point A and Point B.

Figure 10 shows the angular variation of the blue and semi-blue galaxy fraction in the bin of 45 degree. Figure 10 (a) is for all galaxies in Abell 168 except for the $\mathrm{cD}$ galaxy at Point A. Filled and shaded areas are for the blue and the semi-blue galaxies, respectively. Figure 10 (b) is the same as Fig. 10 (a), however, excluding the fainter (> 18.5 mag) objects. A broken line indicates the fraction of the blue and semi-blue galaxies; statistically poor regions (when the total number is less than 9) are neglected. We do not see an enhanced concentration of the blue galaxies along the line between the two x-ray peaks. Figures 10 (c) and (d) are the same as Figs. 10 (a) and (b), respectively, but centered on Point B. Since Point B is near the center of our observed region, angular variation analysis is statistically better. It is much clearer that the angular variation of the blue galaxies is flat. Figure 10 (e) is for the strong Balmer line-absorption galaxies in Coma cluster made from the data by Caldwell et al. (1993). The center is taken to be the x-ray peak in the 
main cluster. The definition of the angle is the same as that for Abell 168 described above. We have selected galaxies in the annular region with radii from the main cluster center from 5 to 48 arcmin; to avoid the very crowded region we have excluded the central region, and to emphasize the distribution in the region between the two x-ray peaks we exclude the outer region. The shaded area is for the strong Balmer-line-absorption galaxies. We can see that the Balmer-line-absorption galaxies frequently exist in the direction of $0^{\circ}$, that is, to the direction of the sub-cluster. We can not see any similar configuration of blue galaxies in Abell 168 as that of the strong Balmer-line-absorption galaxies in the Coma cluster.

There is another important difference between the results presented here and by Caldwell et al. (1993). Since the sample in Caldwell et al. (1993) excluded late-type galaxies, their blue galaxies in Coma are blue early-type galaxies. From Table 4, the number of blue early-type galaxies in Abell 168 is small; our blue galaxies in Abell 168 are mainly blue late-type galaxies. Our sample contains four blue or semi-blue E/S0 galaxies (including S0/Se); No. 18, 44, 97, and 107. They also do not tend to distribute between the two x-ray peaks. Therefore, we found no Coma-phenomenon in Abell 168, though we should note that our search is based only on photometry.

\section{Morphology of the Blue Galaxies}

There are nine galaxies with companions and fifteen double galaxies (for both of them the distance between two galaxies are within 20 arcsec) in our 143 galaxy sample. "Companion" and "double" are distinguished from each other by the size of a companion relative to a galaxy; when the size of the companion is comparable to the galaxy, we call it double. We could not separate objects too close to each other in photometry; in that case, we made photometry including the companion. While none of nine galaxies with 
companions are blue or semi-blue, eight out of fifteen (53\%) double galaxies are blue or semi-blue. Though seeing blurring is serious, we found distorted morphologies in twelve galaxies out of total 143 galaxy sample. Eight out of the distorted galaxies (67 \%) are blue or semi-blue. These two percentages are very high comparing to the total blue-fraction; (23 +17 ) galaxies out of $143(28 \%)$ are blue or semi-blue. Assuming the double or distorted galaxies are in galaxy-galaxy (g-g) interaction, $58 \%$ of the $\mathrm{g}$-g interacting galaxies are blue or semi-blue. Conversely, the blue galaxies tend to show the sign of the g-g interaction; nearly a half of the blue or semi-blue galaxies brighter than $18.5 \mathrm{mag}$ are double or distorted galaxies. Besides, almost all blue or semi-blue galaxies are spirals (see Table 4). In Abell 168, a significant fraction of the blue galaxies are g-g interacting spirals.

\section{Comments on Some Galaxies}

In the following, we give some comments on three dominant cluster members:

No.39: This is an early-spiral and the only $I R A S$ source in our search region. $V=$ $15.2 \mathrm{mag}, I=14.0 \mathrm{mag}$, and $(V-I)=1.2 \mathrm{mag}$. The source name is $I R A S F 01123-0008$ and $f_{60 \mu \mathrm{m}}=0.356 \mathrm{Jy}$. Neither the $4.85 \mathrm{GHz}$ survey by Becker et al. (1991) nor the $1.5 \mathrm{GHz}$ survey by Owen et al. (1993) detected this object. Sopp \& Alexander (1991) showed the

relation between radio $(6 \mathrm{~cm})$ flux and infrared $(60 \mu \mathrm{m})$ flux and found two sequences; one is for radio-quiet galaxies which are considered to be star-forming galaxies $\left(f_{6 \mathrm{~cm}} / f_{60} \mu \mathrm{m}\right.$ $\left.\sim 3 \times 10^{-3}\right)$ and the other is for radio-loud galaxies $\left(f_{6 \mathrm{~cm}} / f_{60 \mu \mathrm{m}} \geq 10\right)$. The radio flux of galaxy No. 39 would be larger than about 3.5 Jy if it is in the radio-loud sequence, which should have been detected in the radio continuum surveys mentioned above. Therefore, galaxy No. 39 is a normal star-forming galaxy.

Assuming that the far-infrared emission of galaxy No. 39 originates from the star- 
forming activity, we can calculate the present star formation rate. Kennicutt et al. (1994) derived the relation between the present star formation rate (SFR) and the $\mathrm{H} \alpha$ luminosity: $\mathrm{SFR}\left[M_{\odot} \mathrm{yr}^{-1}\right] / L(\mathrm{H} \alpha)\left[10^{41} \mathrm{erg} \mathrm{s}^{-1}\right]=1.36$. Young et al. (1989) found the relation between the infrared luminosity and the $\mathrm{H} \alpha$ luminosity: $L_{\mathrm{IR}}(1-500 \mu \mathrm{m})=3.0 \times 10^{2} L(\mathrm{H} \alpha$ ). These relations and $L_{\mathrm{IR}}(1-500 \mu \mathrm{m}) / L_{\mathrm{FIR}}(42.5-122.5 \mu \mathrm{m})=1.5$ for the $(60-100 \mu \mathrm{m})$ color of this object (Cataloged Galaxies and Quasars in the IRAS Survey 1985) lead to a present $\mathrm{SFR}=1.9 M_{\odot} \mathrm{yr}^{-1}$ for this object. The present SFRs in other members are, therefore, lower than about $2 M_{\odot} \mathrm{yr}^{-1}$.

No.46: This is a cD galaxy, the brightest member; $V=13.9 \mathrm{mag}, I=12.6 \mathrm{mag}$, and $(V-I)=1.3 \mathrm{mag}$. The position of this galaxy corresponds to the x-ray peak, the Point A. This galaxy is not detected in $I R A S$ survey nor 4.85 and $1.5 \mathrm{GHz}$ radio continuum surveys mentioned above.

No.81: This is an elliptical and the second brightest member; $V=15.0$ mag, $I=$ $13.7 \mathrm{mag}$, and $(V-I)=1.3 \mathrm{mag}$. Its position is near to the second x-ray peak, the Point B. This is the only radio source detected so far in our searched region; $S(4.85 \mathrm{GHz})=42 \mathrm{mJy}$ (Becker et al. 1991) and $S(1.5 \mathrm{GHz})=49 \mathrm{mJy}$ (Owen et al. 1993). The spectral index of $\alpha$ $\left(F_{\nu} \propto \nu^{\alpha}\right)$ is -0.13 , which indicates a fairly flat spectrum. Assuming $(B-V)=1.0 \mathrm{mag}, R$ $\equiv S(6 \mathrm{~cm}) / S(4400 \AA)=23$ (the definition of $R$ is from Kellermann et al. 1989) this galaxy is in the mid point between radio-loud and radio-quiet objects. If the radio flux of this galaxy is from star-forming activity only, $f_{60 \mu \mathrm{m}}$ would be about $10 \mathrm{Jy}$ and this is bright enough to be detected in the IRAS survey. If the radio flux is from some other powerful activities, $f_{60 \mu \mathrm{m}}$ would be fainter than several mJy and thus be too faint to detect in the $I R A S$ survey. Probably this galaxy belongs to the radio-loud objects.

Many of the spirals in Abell 168 are located near or north of the second brightest member, No. 81, Point B. This distribution is similar to that of sub-cluster members after 
merging in the simulation of Burns et al. (1994); it may be an indirect evidence of the merger event.

\section{Discussion}

In Abell 168 we did not find a concentration of blue galaxies at any place, particularly not in the region between the two x-ray peaks. Though our search is based on photometry only, we could not find the Coma-phenomenon in Abell 168. There are some possibilities to explain our results as follows:

1. almost all infalling galaxies were gas-poor, therefore, they did not have the ability to burst,

2. the orbits on which the ICM pressure increases are not realized,

3. we did not pick up all post-starburst (strong Balmer-line-absorption) galaxies, or

4. star-forming activity is not induced by running into the dense ICM regions.

The hypothesis suggested by Dressler \& Gunn (1983) needs both the gas-rich galaxies and the running orbits toward the dense ICM regions. If almost all galaxies had been gas-poor, blue galaxies could not appear. There is no H I or CO data for Abell 168 members so far, therefore, we do not know whether these galaxies are really gas-poor, or whether they are gas-rich galaxies which do not show strong star-forming activity. Neutral or molecular gas observations are necessary to check this. However, Abell 168 is a spiral-rich cluster and it is improbable that almost all members are gas-poor.

The second possibility is that the orbits may not be realized in Abell 168 because of a lack of the effective ICM compression. The x-ray contrast of two sub-clusters in Abell 168 is about one third of that in Coma, as mentioned in Sec. 5. This may cause much calmer 
environmental change for merging members in Abell 168 than for those in Coma. To clarify the environmental history of member galaxies, high-quality x-ray hot gas observations are necessary.

The strong Balmer-line-absorption galaxies in the Coma cluster studied by Caldwell et al. (1993) are considered to be in the post-starburst phase, however, some of them have already entered into a evolved stage as "weak" Balmer-line-absorption systems. Though most of the blue galaxies (bluer than $\Delta(B-V) \sim 0.1$ mag after correcting the C-M effect) in Coma are the post-starburst galaxies, about one third of all the post-starburst galaxies are not that blue. We may miss some of the post-starburst galaxies in Abell 168. Dressler \& Gunn (1992) investigated the color distribution of emission galaxies, post-starburst galaxies, and passive galaxies in seven rich clusters. From their Fig. 22, generally the post-starburst galaxies (strong Balmer-line-absorption galaxies) have blue colors, however, some of them are as red as passive, typical E/S0 galaxies. In order to pick up the complete post-starburst galaxy population, it is necessary to make spectroscopic observations.

The final possibility might be the case in Abell 168. Our results probably show that the rapid increase of ICM pressure for the galaxies does not work for starburst trigger in Abell 168. However, here we observed only one cluster and we have to check in many other merging clusters. At present we can not say whether our results show that galaxy-ICM interaction does not affect starburst triggering or whether they are due to other causes.

\section{Summary}

We examined whether the first infall model is valid for triggering starbursts in cluster members or not; the model is only satisfied when all of the following three conditions exist: (1) gas-rich galaxies, 
(2) running orbit into the dense ICM regions resulting in the rapid increase of the "external" pressure for the galaxies, and

(3) starburst triggering by compression of molecular clouds by a raised "external" pressure.

The coma-phenomenon, post-starburst galaxies spread between two x-ray peaks, can be interpreted as the result of a cluster merger. Some galaxies would be rushing into the dense ICM regions in the merging cluster. We analyzed the distribution of the blue galaxies, regarded as the star-forming galaxies, in the young merging cluster of galaxies, Abell 168 to see if there is a second example of the Coma-phenomenon. However, we could not find a blue galaxy distribution in Abell 168 to support the model mentioned above. Probably the mechanism does not work effectively as a starburst trigger (negation of (3)), however, there remain some other possibilities. For instance, galaxies may have been already gas-free (contradicting (1)), the orbits on which the ICM pressure increases are not realized (contradicting (2)), or we have missed some post-starburst galaxies which are not blue, and not identified as such in our search. We need cold and hot X-ray gas observations as well as spectroscopic observations, and much data from many other merging clusters for further study. Some starburst galaxies may have left evidences for the galaxy-ICM interaction like metal-enriched "islands" in the hot gas around the galaxies. High spatial-resolution X-ray observations could be another effective way to search for burst-induced galaxies.

We also found in Abell 168 that nearly half of the blue and semi-blue galaxies are spirals in galaxy-galaxy interaction.

We would like to thank Mamoru Saitō, Rainer Spurzem, Takashi Ichikawa, Shin Mineshige, and Chiharu Ishizaka for valuable discussions, as well as the Kiso Observatory staff members for their help in the observations. We also thank to Masaru Hamabe and Nobunari Kashikawa for giving us the latest version of SPIRAL and showing some useful 
techniques on distinguishing between galaxy and stellar images, respectively. This research has made use of the NASA/IPAC Extragalactic Database (NED) which is operated by the Jet Propulsion Laboratory, Caltech, under contact with the National Aeronautics and Space Administration. 


\section{REFERENCES}

Abell, G. O., Corwin, H. G. Jr., \& Olowin, R. P. 1989, ApJS, 70, 1

Arimoto, N., \& Yoshii, Y. 1986, A\&A, 164, 260

Becker, R. H., White, R. L., \& Edwards, A. L. 1991, ApJS, 75, 1

Bica, E., Alloin, D., \& Schmidt, A. 1990, MNRAS, 242, 241

Bothun, G. D., \& Dressler, A. 1986, ApJ, 301, 57

Burns, J. O., Roettiger, K., Ledlow, M., \& Klypin, A. 1994, ApJL, 427, L87

Butcher, H., \& Oemler, A. Jr 1978, ApJ, 219, 18

Butcher, H., \& Oemler, A. Jr 1984, ApJ, 285, 426

Caldwell, N., Rose, J. A., Sharples, R. M., Ellis, R. S., \& Bower, R. G. 1993, AJ, 106, 473

Cataloged Galaxies and Quasars in the IRAS Survey 1985, Lonsdale, C. J., Helou, G., Good, J. C., \& Rice, W., Jet Propulsion Laboratory

Chapmann, G. N. F., Geller, M. J., \& Huchra, J. P. 1988, AJ, 95, 999

Couch, W. J., \& Sharples, R. M. 1987, MNRAS, 229, 423

Cousins, A. W. F. 1976, MemRAS, 81, 25

David, L. P., Slyz, A., Jones, C., Forman, W., Vrtilek, S. D., \& Arnaud, K. A. 1993, ApJ, 412,479

Dressler, A. 1978, ApJ, 223, 765

Dressler, A. 1980, ApJS, 42, 565

Dressler, A., \& Gunn, J. E. 1982, ApJ, 263, 533

Dressler, A., \& Gunn, J. E. 1983, ApJ, 270, 7

Dressler, A., Gunn, J. E., \& Schneider, D. P. 1985, ApJ, 294, 70 
Dressler, A., \& Gunn, J. E. 1988, in Large Scale Structures of the Universe, IAU Symp. No.130, eds. J. Audouze et al. (Kluwer, Dordrecht), p. 311

Dressler, A., \& Gunn, J. E. 1992, ApJS, 78, 1

Einstein Observatory Catalog of IPC X-ray Sources, Volume 1 1990, prepared by The Einstein Catalog Committee (Smithonian Astrophysical Observatory, Cambridge)

Ellis, R. S., Couch, W. J., MacLaren, I., \& Koo, D. C. 1985, MNRAS, 217, 239

Evrard, A. E. 1991, MNRAS, 248, 8p

Faber, S. M., \& Dressler, A. 1977, AJ, 82, 187

Fabricant, D. G., McClintock, J. E., \& Bautz, M. W. 1991, ApJ, 381, 33

Geller, M. J., \& Beers, T. C. 1982, PASP, 94, 421

Gunn, J. E. 1989, in Epoch of Galaxy Evolution, eds. C. S. Frank et al. (Dordrecht: Kluwer), p. 167

Hamabe, M., \& Ichikawa, S. 1992, in Proc. of Astronomical Data Analysis Software and System I, ASPC, 25, eds. D. Worrall et al. (ASP, San Francisco), p. 325

Henry, J. P., \& Lavery, R. J. 1987, ApJ, 323, 473

IRAS Faint Source Catalog 1990, prepared by Joint IRAS Science Working Group

Kellermann, K. I., Sramek, R., Schmidt, M., Shaffer, D. B., \& Green, R. 1989, AJ, 98, 1195

Kennicutt, R. C., Jr., Tamblyn, P., \& Congdon, C. W. 1994, ApJ, 435, 22

Landolt, A. U. 1992, AJ, 104, 340

Lavery, R. J., \& Henry, J. P. 1986, ApJL, 304, L5

Lavery, R. J., \& Henry, J. P. 1988, ApJ, 330, 596

Lavery, R. J., \& Henry, J. P. 1994 ApJ, 426, 524

Lavery, R. J., Pierce, M. J., \& McClure, R. D. 1992, AJ, 104, 2067 
Leitherer, C., \& Heckman, T. M. 1995, ApJS, 96, 9

MacLaren, I., Ellis, R. S., \& Couch, W. J. 1988, MNRAS, 230, 249

Mellier, Y., Soucail, G., Fort, B., \& Mathez, G. 1988, A\&A, 199, 13

Newberry, M. V., Kirshner, R. P., \& Boroson, T. A. 1988, ApJ, 335, 629

Oegerle, W. R., Hoessel, J. G., \& Ernst, R. M. 1986, AJ, 91, 697

Oemler, A. Jr. 1974, ApJ, 194, 1

Owen, F. N., White, R. A., \& Ge, J. P. 1993, ApJS, 87, 135

Sopp, H. M., \& Alexander, P. 1991, MNRAS, 251, 14p

Struble, M. F., \& Rood, H. J. 1982, AJ, 87, 7

Ulmer, M. P., Wirth, G. D., \& Kowalski, M. P. 1992, ApJ, 397, 430

Vigroux, L., \& Boulade, O. 1989, AJ, 98, 2044

Young, J. S., Xie S., Kenney, J. D. P., \& Rice, W. L. 1989, ApJS, 70, 699

Zabludoff, A. I., Geller, M. J., Huchra, J. P., \& Vogeley, M. S. 1993, AJ, 106, 1273

Zwicky, F., Karpowicz, M., \& Kowal C. T. 1965, Catalogue of Galaxies and of Clusters of Galaxies, Volume 5 (California Institute of Technology, Pasadena) 


\section{FIGURE CAPTIONS}

Fig. 1.- Our search region in Abell 168, superimposed on Fig. 2 in Ulmer et al. (1992). Open circles indicate bright galaxies identified by Dressler (1980); their ID-numbers are given

in the ninth column of Table 3, and contours are for x-ray brightness isophotes observed by Einstein IPC $(0.15$ - $4.0 \mathrm{keV})$. A filled square and a filled triangle denote the x-ray peak and the center of galaxy distribution by Ulmer et al. (1992), respectively.

Fig. 2. - The error of photometry for (a) $V$ band data and (b) $I$ band data estimated by comparing data for a given object taken in more than two frames. We show the root-meansquare of measurements for the errors in 1 mag bin. Most of the measurements have an error of less than 0.05 mag.

Fig. 3.- An example of Diagram A, peak count of the image versus FWHM of the image size diagram. This diagram is for the field No. 7 in $V$ band. Open and filled circles show images which are identified by eye-inspection as galaxies and stars, respectively. Crosses indicate the images which are difficult to be discriminated between galaxies and stars.

Fig. 4.- The same as Fig. 3 but for (peak count)/FWHM ${ }^{2}$ versus magnitude diagram (Diagram B).

Fig. 5.- The differential luminosity function of Abell 168 galaxies. Numbers are normalized to what is expected to be in $0.2 \mathrm{deg}^{2}$. Filled circles represent our sample. Note that our sample contains the background contamination. Open squares and open triangles show Dressler (1978) and Oegerle et al. (1986) sample, respectively. A solid line shows field galaxy count by Oemler (1974), which was used for the background-contamination correction in Dressler (1978) and Oegerle et al. (1986).

Fig. 6.- $(V-I)$ color - $V$ magnitude diagram for Abell 168 galaxies (C-M diagram). A solid line shows the typical color for early-type galaxies, the C-M effect. The blue and semi-blue objects are shown by the filled and shaded circles, respectively. 
Fig. 7.- $(V-I)_{\text {corr }}$ color distribution after correcting the C-M effect. Filled and shaded regions correspond to the blue and semi-blue objects, respectively. We choose the color of $(V-I)_{\text {corr }}=1.2 \mathrm{mag}$ to be peak of the color, shown by a dashed line. Note that this color corresponds to the solid line in Fig. 5.

Fig. 8. - Distribution of all galaxies and blue galaxies detected in our survey. The blue and semi-blue galaxies are shown by the filled and shaded circles, respectively. The ID-numbers are given for the blue and semi-blue objects and some other interesting objects mentioned in text. The numbers with circles are for the objects brighter than 18.5 mag. The size of circles is proportional to the size of major axis, tabulated in the third column of Table 3. Note that the size scale is expanded twice compared to the galaxy separation scale for comprehension. Dashed curves indicate the x-ray (0.15-4.0 keV) contours by Ulmer et al. (1992). A cross indicates the cluster center used for Fig. 10, the maximum of galaxy surface number density by Geller \& Beers (1982).

Fig. 9.- The fraction of blue and semi-blue galaxies with the cluster-centric radius for (a) all 143 galaxies and for (b) only the brighter $(\leq 18.5 \mathrm{mag}) 109$ galaxies. The center is taken to be the maximum of the galaxy surface number density by Geller \& Beers (1982), shown by the cross in Fig. 7; this point is located near the center of our search region. A broken line indicates the fraction of the blue and semi-blue galaxies; the scale is shown on the right side. Note that we searched only in the central region of the cluster. The Abell radius $\left(R_{\mathrm{A}}\right.$ [arcmin] $=1.7 z^{-1}$ ) of Abell 168 is 38 arcmin. The blue and semi-blue galaxies tend to avoid the central region with a radius of $0.1 R_{\mathrm{A}}$. Though the statistical error is large, the blue and semi-blue galaxies seem to concentrate within $0.4 R_{\mathrm{A}}$. 
Fig. 10.- The angular variation of the fraction of the blue galaxies. (a) For the blue and semi-blue galaxies in Abell 168 from our data. Center is taken to be Point A. Filled area is for the blue galaxies and shaded area is for the semi-blue galaxies. A broken line indicates the fraction of the blue and semi-blue galaxies; the scale is shown on the right side. If the total number in a cone region is less than 9, we omit calculation of the fraction in that division because of poor statistics. No significant over-distribution of the blue galaxies is seen in the angle of $0^{\circ}$. (b) The same as (a), however, excluding the fainter (>18.5 mag) objects. Again we can not see the over-distribution of the blue and semi-blue galaxies in the angle of $0^{\circ}$. (c)(d) The same as (a)(b), however, centered on Point B. The flatness of the angular variation of the blue galaxies is seen much clearly. (e) For the strong Balmer-line-absorption galaxies in Coma cluster; the data are taken from Caldwell et al. (1993). Shaded area is for the strong Balmer-line-absorption galaxies. Enhanced distribution in the angle of $0^{\circ}$, the direction to the sub-cluster is seen clearly. 


\section{TABLE CAPTIONS}

TABLE 1. Characteristics of Abell 168.

TABLE 2. A journal of observations.

TABLE 3. Catalog of galaxies in Abell 168.

TABLE 4. Distribution of the morphology. 
TABLE 1. Characteristics of Abell 168.

\begin{tabular}{|c|c|c|c|}
\hline \multirow[t]{6}{*}{$\overline{\text { Center }}$} & $\alpha(1950.0)$ & $01^{\mathrm{h}} 12^{\mathrm{m}} \cdot 6$ & $(1)$ \\
\hline & $\delta(1950.0)$ & $-00^{\circ} 01^{\prime}$ & (1) \\
\hline & $\alpha(2000.0)$ & $01^{\mathrm{h}} 15^{\mathrm{m}} .2$ & (1) \\
\hline & $\delta(2000.0)$ & $00^{\circ} 14^{\prime}$ & (1) \\
\hline & $l$ & $135 .^{\circ} 67$ & (1) \\
\hline & $b$ & $-62 .^{\circ} 04$ & (1) \\
\hline \multirow[t]{2}{*}{ Type } & Bautz-Morgan & II-III: & (1) \\
\hline & Rood-Sastry & $\mathrm{I}_{\mathrm{C}}$ & $(2)$ \\
\hline \multicolumn{2}{|l|}{ Count $^{\mathrm{a}}$} & 89 & (1) \\
\hline \multicolumn{2}{|c|}{ Radial velocity } & $13428 \mathrm{~km} \mathrm{~s}^{-1}$ & (3) \\
\hline \multicolumn{2}{|c|}{ Richness class } & 2 & (1) \\
\hline \multicolumn{2}{|c|}{ Distance class } & 3 & (1) \\
\hline \multicolumn{2}{|c|}{$m_{10}^{\mathrm{b}}$} & 15.4 & (1) \\
\hline \multicolumn{2}{|c|}{ Radial velocity dispersion } & $461 \mathrm{~km} \mathrm{~s}^{-1}$ & (3) \\
\hline \multicolumn{2}{|c|}{ Diameter of the cluster } & $3 \mathrm{Mpc}$ & (5) \\
\hline \multicolumn{2}{|c|}{ Mass of the cluster ${ }^{c}$} & $3.5 \times 10^{14} M_{\odot}$ & \\
\hline \multirow{2}{*}{\multicolumn{2}{|c|}{$\begin{array}{l}\text { Temperature of the hot gas } \\
\text { Peak X-ray surface brightness }\end{array}$}} & $3.0 \times 10^{7} \mathrm{~K}(2.6 \mathrm{keV})$ & (4) \\
\hline & & & \\
\hline & Point A & $9 \times 10^{-14} \mathrm{erg} \mathrm{s}^{-1} \mathrm{~cm}^{-2} \operatorname{arcmin}^{-2}$ & \\
\hline & Point B & $3 \times 10^{-14} \mathrm{erg} \mathrm{s}^{-1} \mathrm{~cm}^{-2} \operatorname{arcmin}^{-2}$ & \\
\hline
\end{tabular}

Notes to TABLE 1

${ }^{a}$ Background-corrected count of cluster members in the magnitude range $m_{3}$ to $m_{3}+2$, where $m_{2}$ is the magnitude of the third brightest galaxy.

$\mathrm{b}$ The red magnitude of the tenth brightest cluster member.

c The cluster mass $M$ is derived from the equation, $M=3 R \sigma_{\text {gal }}^{2} / G . R$ is the diameter of the cluster and $\sigma_{\text {gal }}$ is the radial velocity dispersion of the galaxies; both values are taken from those tabulated here.

${ }^{\mathrm{d}}$ Measured by Einstein IPC $(0.15-4.0 \mathrm{keV})$. We referred to Einstein Observatory Catalog of IPC X-ray Sources Volume 1 (1990).

References

(1) Abell et al. (1989)

(2) Struble \& Rood (1982)

(3) Zabludoff et al. (1993)

(4) David et al. (1993)

(5) Zwicky, Karpowicz, \& Kowal (1965) 
TABLE 2. A journal of observations.

\begin{tabular}{|c|c|c|c|c|}
\hline No. & $\begin{array}{c}\text { Field center } \\
\alpha(1950.0) \delta\end{array}$ & $\begin{array}{l}\text { Total exposure } \\
\text { time (sec) }\end{array}$ & $\begin{array}{c}\text { Observation } \\
\text { Date }\end{array}$ & $\begin{array}{r}\text { Seeing size } \\
(\operatorname{arcsec})^{\mathrm{a}}\end{array}$ \\
\hline & $\begin{array}{lllll}\mathrm{h} & \mathrm{m} & \mathrm{s} & \circ & \mathrm{l}\end{array}$ & $(V, I)$ & $(1993)$ & $(V, I)$ \\
\hline 1 & 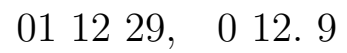 & 300,300 & 15 November & $3.7,3.2$ \\
\hline 2 & 0112 28, 005.6 & 300,300 & 15 November & $3.8,3.5$ \\
\hline 3 & $011301, \quad 005.3$ & 1500,1500 & 15 November & $3.5,4.1$ \\
\hline 4 & $011301,-002.3$ & 2700,3300 & 08 December & $3.1,3.0$ \\
\hline 5 & $011158, \quad 012.9$ & 2100,2100 & 09 December & $4.2,4.2$ \\
\hline 6 & $011302,-006.6$ & 1200,1200 & 12 December & $2.9,2.9$ \\
\hline 7 & $011228,-002.3$ & 2700,2100 & 12 December & $3.1,2.9$ \\
\hline
\end{tabular}

Notes to TABLE 2

${ }^{a}$ FWHM of stellar images in the reduced frames. 
TABLE 3. Catalog of galaxies in Abell 168.

\begin{tabular}{|c|c|c|c|c|c|c|c|c|c|c|}
\hline No. & \multicolumn{2}{|c|}{$\begin{array}{c}\text { Position } \\
\alpha(1950.0) \delta\end{array}$} & \multirow{2}{*}{$\begin{array}{r}\text { Size } \\
(4) \\
\end{array}$} & \multirow{2}{*}{$\begin{array}{l}V \\
(5)\end{array}$} & \multirow{2}{*}{$\begin{array}{c}I \\
(6) \\
\end{array}$} & \multirow{2}{*}{$\begin{array}{c}(V-I) \\
(7) \\
\end{array}$} & \multirow{2}{*}{$\begin{array}{c}\text { Morp. } \\
(8) \\
\end{array}$} & \multirow{2}{*}{$\begin{array}{l}\text { D80 } \\
\text { No. } \\
(9) \\
\end{array}$} & \multirow{2}{*}{$\begin{array}{c}\text { Radial } \\
\text { velocity } \\
(10)\end{array}$} & \multirow{2}{*}{$\begin{array}{r}\text { Notes } \\
\text { (11) }\end{array}$} \\
\hline (1) & $(2)$ & (3) & & & & & & & & \\
\hline & $\begin{array}{lll}\mathrm{h} & \mathrm{m} & \mathrm{s} \\
\end{array}$ & 01 & {$\left[{ }^{\prime \prime}\right]$} & & & & & & {$\left[\mathrm{km} \mathrm{s}^{-1}\right]$} & \\
\hline 1 & 11139 & 017.1 & 5 & 18.8 & 17.8 & 1.0 & $\mathrm{Se}$ & & & $\mathrm{sb}$ \\
\hline 2 & 11142 & 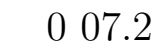 & 11 & 17.5 & 16.3 & 1.2 & $\mathrm{E}$ & & & $\mathrm{COM}$ \\
\hline 3 & 11147 & 012.9 & 19 & 16.9 & 15.8 & 1.2 & $\mathrm{E} / \mathrm{S} 0$ & 94 & & \\
\hline 4 & 11148 & 008.7 & 6 & 18.8 & 17.5 & 1.3 & $\mathrm{Se}$ & & & \\
\hline 5 & 11148 & 009.3 & 21 & 16.3 & 15.1 & 1.2 & $\mathrm{E}$ & 84 & & \\
\hline 6 & 11149 & 016.7 & 33 & 15.2 & 14.2 & 0.9 & $\mathrm{Se}$ & 102 & 12486 & b, b-disk \\
\hline 7 & 11153 & 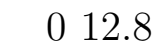 & 16 & 16.8 & 15.6 & 1.3 & $\mathrm{E}$ & 93 & & \\
\hline 8 & 11153 & 015.5 & 10 & 17.8 & 16.7 & 1.1 & $\mathrm{Se}$ ? & & & COM? \\
\hline 9 & 11154 & 016.2 & 17 & 16.7 & 15.6 & 1.2 & $\mathrm{E} / \mathrm{S} 0$ & 101 & & \\
\hline 10 & 11155 & 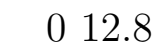 & 11 & 17.7 & 16.4 & 1.3 & $\mathrm{E}$ & & & \\
\hline 11 & 11155 & 013.1 & 8 & 19.1 & 17.5 & 1.6 & $\mathrm{Se}$ & & & \\
\hline 12 & 11156 & 012.7 & 10 & 18.2 & 17.0 & 1.2 & $\mathrm{E}$ & & & DBL \\
\hline 13 & 11156 & 016.3 & 21 & 16.3 & 15.1 & 1.2 & S0 & 100 & & \\
\hline 14 & 11200 & $\begin{array}{ll}0 & 12.9\end{array}$ & 9 & 17.7 & 16.6 & 1.1 & $\mathrm{E}$ & & & \\
\hline 15 & 11204 & 001.1 & 5 & 18.7 & 17.8 & 0.9 & $\mathrm{Se} / \mathrm{Sl}$ & & & $\mathrm{b}$ \\
\hline 16 & 11204 & 010.6 & 3 & 19.0 & 18.4 & 0.6 & $\mathrm{Se} / \mathrm{I}$ & & & LSB, DBL(17), b \\
\hline 17 & 11205 & $\begin{array}{l}0 \\
0\end{array}$ & 8 & 18.5 & 17.6 & 0.9 & $\mathrm{Se}$ & & & $\operatorname{DBL}(16), \mathrm{b}$ \\
\hline 18 & 11205 & 012.5 & 23 & 16.1 & 15.0 & 1.1 & So & 92 & 13360 & $\mathrm{sb}$ \\
\hline 19 & 11208 & -007.6 & 17 & 17.0 & 15.8 & 1.2 & So & 42 & & barred? \\
\hline 20 & 11208 & -000.6 & 13 & 18.0 & 16.9 & 1.1 & $\mathrm{Se}$ & & & \\
\hline 21 & 11209 & 001.9 & 29 & 15.8 & 14.7 & 1.1 & $\mathrm{Se}$ & 72 & & dist, sb \\
\hline 22 & 11210 & $\begin{array}{ll}0 & 17.3\end{array}$ & 7 & 18.9 & 17.7 & 1.2 & $\mathrm{Se} / \mathrm{I}$ & & & \\
\hline 23 & 11211 & 016.3 & 8 & 18.2 & 17.1 & 1.1 & $\mathrm{Se} ?$ & & & \\
\hline 24 & 11212 & 009.8 & 7 & 19.0 & 17.7 & 1.3 & $?$ & & & red \\
\hline 25 & 11212 & 013.4 & 7 & 16.0 & 14.7 & 1.4 & $?$ & & & DBL \\
\hline 26 & 11213 & 015.6 & 34 & 15.4 & 14.1 & 1.3 & $\mathrm{Se}$ & 99 & 13597 & r-circum-nucleus \\
\hline 27 & 11213 & 018.7 & 11 & 18.1 & 16.7 & 1.4 & $\mathrm{SB} e$ & & & r-bar \\
\hline 28 & 11214 & -001.7 & 22 & 16.1 & 14.9 & 1.2 & S0 & 71 & 13975 & COM? \\
\hline 29 & 11214 & 004.0 & 9 & 18.4 & 17.6 & 0.8 & $\mathrm{Se}$ & & & dist, b \\
\hline 30 & 11217 & 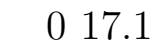 & 5 & 17.0 & 15.9 & 1.2 & $\mathrm{Se}$ & 98 & & \\
\hline
\end{tabular}


TABLE 3. (continued)

\begin{tabular}{|c|c|c|c|c|c|c|c|c|c|c|}
\hline \multirow{2}{*}{$\begin{array}{l}\text { No. } \\
\text { (1) }\end{array}$} & \multicolumn{2}{|c|}{$\begin{array}{c}\text { Position } \\
\alpha(1950.0) \delta\end{array}$} & \multirow{2}{*}{$\begin{array}{l}\text { Size } \\
(4)\end{array}$} & \multirow{2}{*}{$\begin{array}{l}V \\
(5)\end{array}$} & \multirow{2}{*}{$\begin{array}{l}I \\
(6)\end{array}$} & \multirow{2}{*}{$\begin{array}{c}(V-I) \\
(7)\end{array}$} & \multirow{2}{*}{$\begin{array}{l}\text { Morp. } \\
\text { (8) }\end{array}$} & \multirow{2}{*}{$\begin{array}{l}\text { D80 } \\
\text { No. } \\
(9)\end{array}$} & \multirow{2}{*}{$\begin{array}{c}\text { Radial } \\
\text { velocity } \\
(10)\end{array}$} & \multirow{2}{*}{$\begin{array}{r}\text { Notes } \\
\text { (11) }\end{array}$} \\
\hline & $(2)$ & (3) & & & & & & & & \\
\hline & $\begin{array}{lll}\mathrm{h} & \mathrm{m} & \mathrm{s}\end{array}$ & 01 & {$\left[{ }^{\prime \prime}\right]$} & & & & & & {$\left[\mathrm{km} \mathrm{s}^{-1}\right]$} & \\
\hline 31 & 11219 & 000.6 & 7 & 18.4 & 17.1 & 1.2 & $\mathrm{Se}$ & & & \\
\hline 32 & 11219 & 002.8 & 28 & 15.2 & 13.9 & 1.3 & $\mathrm{E}$ & 70 & 13294 & \\
\hline 33 & 11219 & 007.8 & 7 & 18.6 & 17.5 & 1.1 & $\mathrm{Se}$ & & & \\
\hline 34 & 11220 & 014.5 & 25 & 15.7 & 14.5 & 1.3 & $\mathrm{Se}$ & 91 & & r-bar? \\
\hline 35 & 11221 & 002.2 & 12 & 17.4 & 16.2 & 1.1 & $\mathrm{~S} 0 / \mathrm{Se}$ & 69 & & \\
\hline 36 & 11221 & 012.7 & 13 & 17.5 & 16.3 & 1.2 & $\mathrm{E} / \mathrm{S} 0$ & & & dist \\
\hline 37 & 11222 & 002.5 & 8 & 19.0 & 17.6 & 1.4 & $\mathrm{Se}$ & & & \\
\hline 38 & 11222 & 007.8 & 15 & 16.9 & 15.6 & 1.2 & $\mathrm{E}$ & 83 & & \\
\hline 39 & 11223 & -008.0 & 31 & 15.2 & 14.0 & 1.2 & $\mathrm{Se}$ & 41 & 12485 & r-bar \\
\hline 40 & 11223 & -003.4 & 8 & 18.7 & 18.2 & 0.6 & $\mathrm{I} ?$ & & & $\operatorname{DBL}(47), \mathrm{b}$ \\
\hline 41 & 11223 & 010.6 & 13 & 17.8 & 16.4 & 1.4 & $\mathrm{E}$ & & & \\
\hline 42 & 11223 & $\begin{array}{lll}0 & 16.0\end{array}$ & 13 & 17.4 & 16.2 & 1.2 & $\mathrm{E}$ & & & \\
\hline 43 & 11224 & -006.9 & 8 & 18.5 & 17.2 & 1.3 & $\mathrm{Se}$ & & & \\
\hline 44 & 11224 & -006.1 & 8 & 18.6 & 17.6 & 1.0 & $\mathrm{~S} 0 / \mathrm{Se}$ & & & $\mathrm{sb}$ \\
\hline 45 & 11224 & 000.3 & 11 & 17.5 & 16.3 & 1.2 & E & & & \\
\hline 46 & 11224 & 010.0 & 55 & 13.9 & 12.6 & 1.3 & $\mathrm{E}(\mathrm{cD})$ & 90 & 13439 & \\
\hline 47 & 11225 & -003.3 & 8 & 17.9 & 17.2 & 0.7 & $\mathrm{Se}$ & & & $\operatorname{DBL}(40), b$ \\
\hline 48 & 11225 & -000.8 & 8 & 18.5 & 17.5 & 1.0 & $\mathrm{Se} ?$ & & & $\mathrm{sb}$ \\
\hline 49 & 11225 & 011.6 & 8 & 18.7 & 17.4 & 1.3 & $\mathrm{Se}$ & & & \\
\hline 50 & 11226 & -007.3 & 14 & 17.6 & 16.3 & 1.4 & $\mathrm{Se}$ & & & \\
\hline 51 & 11226 & -002.8 & 11 & 18.0 & 16.9 & 1.1 & $\mathrm{Se}$ & & & \\
\hline 52 & 11226 & -000.2 & 28 & 15.4 & 14.1 & 1.3 & $\mathrm{SB} e$ & 68 & 14540 & r-bar \\
\hline 53 & 11226 & 006.5 & 10 & 18.5 & 17.2 & 1.3 & $?$ & & & \\
\hline 54 & 11226 & 015.3 & 7 & 18.9 & 17.8 & 1.0 & $?$ & & & \\
\hline 55 & 11228 & 000.2 & 23 & 16.4 & 15.2 & 1.2 & $\mathrm{Se}$ & 66 & & \\
\hline 56 & 11228 & 002.9 & 21 & 16.7 & 15.5 & 1.2 & $\mathrm{Se}$ & 67 & & \\
\hline 57 & 11230 & 008.4 & 32 & 15.0 & 13.8 & 1.2 & $\mathrm{Se}$ & 82 & 13340 & \\
\hline 58 & 11230 & 017.3 & 8 & 19.0 & 17.7 & 1.3 & $\mathrm{I} ?$ & & & \\
\hline 59 & 11231 & 003.7 & 31 & 15.7 & 14.4 & 1.3 & $\mathrm{Se}$ & 81 & 12625 & \\
\hline 60 & 11232 & -000.9 & 10 & 18.5 & 17.3 & 1.2 & $\mathrm{Se}$ & & & \\
\hline
\end{tabular}


TABLE 3. (continued)

\begin{tabular}{|c|c|c|c|c|c|c|c|c|c|c|}
\hline \multirow{2}{*}{$\begin{array}{l}\text { No. } \\
\text { (1) }\end{array}$} & \multicolumn{2}{|c|}{$\begin{array}{c}\text { Position } \\
\alpha(1950.0) \delta\end{array}$} & \multirow{2}{*}{$\begin{array}{l}\text { Size } \\
\text { (4) }\end{array}$} & \multirow{2}{*}{$\begin{array}{l}V \\
(5)\end{array}$} & \multirow{2}{*}{$\begin{array}{l}I \\
(6)\end{array}$} & \multirow{2}{*}{$\begin{array}{c}(V-I) \\
(7)\end{array}$} & \multirow{2}{*}{$\begin{array}{l}\text { Morp. } \\
\text { (8) }\end{array}$} & \multirow{2}{*}{$\begin{array}{l}\text { D80 } \\
\text { No. } \\
(9)\end{array}$} & \multirow{2}{*}{$\begin{array}{c}\text { Radial } \\
\text { velocity } \\
(10)\end{array}$} & \multirow{2}{*}{$\begin{array}{r}\text { Notes } \\
\text { (11) }\end{array}$} \\
\hline & $(2)$ & (3) & & & & & & & & \\
\hline & $\begin{array}{lll}\mathrm{h} & \mathrm{m} & \mathrm{s} \\
\end{array}$ & 01 & {$\left[{ }^{\prime \prime}\right]$} & & & & & & {$\left[\mathrm{km} \mathrm{s}^{-1}\right]$} & \\
\hline 61 & 11233 & 009.8 & 7 & 18.3 & 17.5 & 0.7 & $?$ & & & $\mathrm{~b}$ \\
\hline 62 & 11234 & -002.8 & 9 & 18.7 & 17.8 & 0.9 & $\mathrm{Se}$ & & & b \\
\hline 63 & 11234 & $\begin{array}{ll}-0 & 02.1\end{array}$ & 23 & 16.4 & 15.3 & 1.1 & $\mathrm{Se}$ & 63 & & dist, sb \\
\hline 64 & 11234 & 011.9 & 27 & 15.6 & 14.3 & 1.3 & $\mathrm{~S} 0 / \mathrm{Se}$ & 89 & 12560 & \\
\hline 65 & 11235 & 000.3 & 22 & 16.0 & 14.8 & 1.2 & E & 62 & & \\
\hline 66 & 11235 & 002.1 & 21 & 16.5 & 15.5 & 1.1 & $\mathrm{Se}$ & 64 & 13658 & $\operatorname{DBL}(67), \mathrm{sb}$ \\
\hline 67 & 11235 & 002.6 & 21 & 16.4 & 15.2 & 1.2 & $\mathrm{Se}$ & 65 & 14027 & $\operatorname{DBL}(66)$, swb \\
\hline 68 & 11236 & 018.2 & 26 & 15.4 & 14.1 & 1.3 & $\mathrm{Se}$ & 97 & & \\
\hline 69 & 11237 & 002.8 & 9 & 18.6 & 17.6 & 1.0 & $\mathrm{Se}$ & & & $\mathrm{sb}$ \\
\hline 70 & 11238 & -006.6 & 7 & 18.6 & 17.5 & 1.1 & $?$ & & & \\
\hline 71 & 11239 & -012.7 & 11 & 18.4 & 17.3 & 1.2 & $\mathrm{E}$ & & & \\
\hline 72 & 11239 & 003.4 & 7 & 18.9 & 17.4 & 1.5 & $\mathrm{Se}$ & & & \\
\hline 73 & 11239 & 018.2 & 9 & 17.8 & 16.7 & 1.1 & $?$ & & & \\
\hline 74 & 11240 & 001.6 & 19 & 16.9 & 15.8 & 1.1 & S0 & 61 & & \\
\hline 75 & 11240 & 004.6 & 21 & 15.9 & 14.6 & 1.3 & $\mathrm{E}$ & 80 & 13453 & \\
\hline 76 & 11241 & -001.4 & 9 & 17.8 & 16.6 & 1.3 & E & & & \\
\hline 77 & 11241 & 004.4 & 20 & 16.4 & 15.3 & 1.1 & $\mathrm{Se} / \mathrm{Sl}$ & 79 & & \\
\hline 78 & 11241 & 013.2 & 12 & 17.6 & 16.7 & 0.9 & $\mathrm{Se}$ & 95 & & $\mathrm{~b}$ \\
\hline 79 & 11242 & -009.1 & 8 & 18.7 & 17.5 & 1.1 & $\mathrm{Se} ?$ & & & \\
\hline 80 & 11242 & -008.6 & 14 & 18.4 & 17.2 & 1.2 & $\mathrm{Se}$ & & & DBL \\
\hline 81 & 11242 & $\begin{array}{ll}-0 & 03.1\end{array}$ & 35 & 15.0 & 13.7 & 1.3 & $\mathrm{E}$ & 40 & 13553 & \\
\hline 82 & 11243 & $\begin{array}{ll}-0 & 10.7\end{array}$ & 22 & 16.4 & 15.3 & 1.1 & $\mathrm{Se}$ & 28 & & dist, sb \\
\hline 83 & 11243 & -006.2 & 18 & 17.1 & 16.1 & 1.0 & $\mathrm{Se}$ & & & dist, b \\
\hline 84 & 11243 & -004.8 & 32 & 15.1 & 13.8 & 1.3 & $\mathrm{E}$ & 38,39 & 13420 & DBL \\
\hline 85 & 11243 & -002.0 & 8 & 18.6 & 17.3 & 1.2 & $?$ & & & \\
\hline 86 & 11243 & -001.7 & 17 & 17.3 & 16.1 & 1.2 & $\mathrm{Se}$ & 60 & & \\
\hline 87 & 11243 & 000.8 & 7 & 18.6 & 17.8 & 0.8 & $\mathrm{Sl} / \mathrm{SB} l$ & & & LSB, b \\
\hline 88 & 11243 & 002.8 & 13 & 17.9 & 17.0 & 0.8 & $\mathrm{~S} e ?$ & & & $\mathrm{~b}$ \\
\hline 89 & 11244 & -010.0 & 15 & 18.4 & 17.6 & 0.8 & $\mathrm{~S} l$ & & & LSB, b \\
\hline 90 & 11244 & -006.0 & 15 & 16.6 & 15.3 & 1.3 & $\mathrm{E}$ & 37 & & \\
\hline
\end{tabular}


TABLE 3. (continued)

\begin{tabular}{|c|c|c|c|c|c|c|c|c|c|c|}
\hline No. & \multicolumn{2}{|c|}{$\begin{array}{c}\text { Position } \\
\alpha(1950.0) \delta\end{array}$} & \multirow{2}{*}{$\begin{array}{l}\text { Size } \\
\text { (4) }\end{array}$} & \multirow{2}{*}{$\begin{array}{l}V \\
(5)\end{array}$} & \multirow{2}{*}{$\begin{array}{l}I \\
(6)\end{array}$} & \multirow{2}{*}{$\begin{array}{c}(V-I) \\
(7)\end{array}$} & \multirow{2}{*}{$\begin{array}{l}\text { Morp. } \\
\text { (8) }\end{array}$} & \multirow{2}{*}{$\begin{array}{l}\text { D80 } \\
\text { No. } \\
(9)\end{array}$} & \multirow{2}{*}{$\begin{array}{c}\text { Radial } \\
\text { velocity } \\
(10)\end{array}$} & \multirow{2}{*}{$\begin{array}{c}\text { Notes } \\
\text { (11) }\end{array}$} \\
\hline (1) & $(2)$ & $(3)$ & & & & & & & & \\
\hline & $\begin{array}{lll}\mathrm{h} & \mathrm{m} & \mathrm{s} \\
\end{array}$ & 01 & {$\left[{ }^{\prime \prime}\right]$} & & & & & & {$\left[\mathrm{km} \mathrm{s}^{-1}\right]$} & \\
\hline 91 & 11244 & $\begin{array}{ll}0 & 06.9\end{array}$ & 10 & 18.3 & 17.1 & 1.2 & $?$ & & & \\
\hline 92 & 11244 & $\begin{array}{lll}0 & 07.9\end{array}$ & 9 & 18.5 & 17.6 & 0.9 & $\mathrm{Se} ?$ & & & b \\
\hline 93 & 11245 & -001.0 & 8 & 18.7 & 17.7 & 1.0 & $\mathrm{Se}$ & & & $\mathrm{sb}$ \\
\hline 94 & 11245 & 000.1 & 6 & 18.6 & 17.4 & 1.1 & $\mathrm{Se}$ & & & COM? \\
\hline 95 & 11246 & -0 01.9 & 13 & 17.4 & 16.2 & 1.3 & $\mathrm{Se}$ & & & dist \\
\hline 96 & 11247 & -000.3 & 40 & 15.5 & 14.2 & 1.2 & $\mathrm{Se}$ & 59 & 13074 & $\mathrm{COM}$ \\
\hline 97 & 11248 & 004.6 & 16 & 17.1 & 16.0 & 1.0 & $\mathrm{E}$ & & & $\mathrm{sb}$ \\
\hline 98 & 11248 & 010.5 & 8 & 18.7 & 17.4 & 1.3 & $\mathrm{~S} l$ & & & \\
\hline 99 & 11248 & 011.1 & 6 & 18.6 & 17.9 & 0.7 & $?$ & & & b \\
\hline 100 & 11250 & $\begin{array}{ll}-0 & 09.1\end{array}$ & 7 & 18.8 & 17.7 & 1.2 & $?$ & & & \\
\hline 101 & 11251 & -007.5 & 6 & 19.0 & 18.0 & 1.0 & $\mathrm{Se}$ & & & $\mathrm{sb}$ \\
\hline 102 & 11251 & -000.7 & 23 & 16.8 & 15.6 & 1.2 & $\mathrm{Se}$ & 58 & & \\
\hline 103 & 11251 & 001.1 & 23 & 16.0 & 14.7 & 1.3 & $\mathrm{E}$ & 57 & 14083 & \\
\hline 104 & 11252 & -011.7 & 19 & 16.6 & 15.4 & 1.2 & S0 & 27 & & \\
\hline 105 & 11252 & 002.8 & 7 & 15.8 & 14.9 & 0.8 & $?$ & & & DBL, b \\
\hline 106 & 11252 & 007.6 & 10 & 18.4 & 17.2 & 1.2 & $\mathrm{~S} 0 / \mathrm{S} e$ & & & \\
\hline 107 & 11253 & -003.5 & 15 & 16.8 & 15.8 & 1.1 & $\mathrm{~S} 0 / \mathrm{Se}$ & 35 & & $\mathrm{sb}$ \\
\hline 108 & 11253 & -003.0 & 12 & 17.4 & 16.1 & 1.3 & E & 36 & & \\
\hline 109 & 11254 & 003.6 & 7 & 18.6 & 17.7 & 0.8 & $\mathrm{Se} ?$ & & & LSB, b \\
\hline 110 & 11256 & -005.6 & 16 & 17.4 & 16.1 & 1.3 & $\mathrm{Se}$ & 34 & & COM, b-comp, dist \\
\hline 111 & 11256 & 004.0 & 11 & 17.9 & 16.8 & 1.1 & E/S0 & & & \\
\hline 112 & 11257 & -002.4 & 26 & 15.6 & 14.4 & 1.2 & E & 56 & & \\
\hline 113 & 11257 & 002.1 & 27 & 15.4 & 14.1 & 1.3 & $\mathrm{E}$ & 55 & 13944 & $\mathrm{COM}$ \\
\hline 114 & 11257 & 002.9 & 15 & 17.1 & 15.8 & 1.3 & $\mathrm{E} / \mathrm{S} 0$ & & & DBL \\
\hline 115 & 11258 & 001.5 & 26 & 15.8 & 14.6 & 1.2 & E & 54 & 14008 & DBL \\
\hline 116 & 11259 & -011.6 & 13 & 17.7 & 16.5 & 1.2 & $\mathrm{Se}$ & & & \\
\hline 117 & 11302 & $\begin{array}{ll}0 & 07.7\end{array}$ & 20 & 17.3 & 16.2 & 1.1 & $\mathrm{~S} 0 / \mathrm{Se}$ & & & dist \\
\hline 118 & 11302 & 010.6 & 8 & 18.7 & 17.5 & 1.1 & $?$ & & & \\
\hline 119 & 11304 & -0 10.4 & 12 & 18.0 & 16.9 & 1.1 & $\mathrm{Se}$ & & & \\
\hline 120 & 11304 & -007.4 & 13 & 18.2 & 17.1 & 1.0 & $\mathrm{Se}$ & & & LSB, DBL, dist, sb \\
\hline
\end{tabular}


TABLE 3. (continued)

\begin{tabular}{|c|c|c|c|c|c|c|c|c|c|c|}
\hline No. & \multicolumn{2}{|c|}{$\begin{array}{c}\text { Position } \\
\alpha(1950.0) \delta\end{array}$} & \multirow{2}{*}{$\begin{array}{r}\text { Size } \\
(4) \\
\end{array}$} & \multirow{2}{*}{$\begin{array}{l}V \\
(5) \\
\end{array}$} & \multirow{2}{*}{$\begin{array}{l}I \\
(6) \\
\end{array}$} & \multirow{2}{*}{$\begin{array}{c}(V-I) \\
(7) \\
\end{array}$} & \multirow{2}{*}{$\begin{array}{l}\text { Morp. } \\
\text { (8) }\end{array}$} & \multirow{2}{*}{$\begin{array}{l}\text { D80 } \\
\text { No. } \\
(9)\end{array}$} & \multirow{2}{*}{$\begin{array}{c}\text { Radial } \\
\text { velocity } \\
(10)\end{array}$} & \multirow{2}{*}{$\begin{array}{l}\text { Notes } \\
\text { (11) }\end{array}$} \\
\hline (1) & $(2)$ & (3) & & & & & & & & \\
\hline & $\begin{array}{ll}\mathrm{h} & \mathrm{m} \\
\end{array}$ & 01 & {$\left[{ }^{\prime \prime}\right]$} & & & & & & {$\left[\mathrm{km} \mathrm{s}^{-1}\right]$} & \\
\hline 121 & 11304 & -002.8 & 10 & 18.3 & 17.1 & 1.2 & $\mathrm{~S} 0 / \mathrm{Se}$ & & & \\
\hline 122 & 11304 & 006.0 & 8 & 18.5 & 17.6 & 1.0 & $\mathrm{Se}$ & & & $\mathrm{sb}$ \\
\hline 123 & 11306 & -009.8 & 20 & 16.6 & 15.4 & 1.2 & $\mathrm{E}$ & 26 & & \\
\hline 124 & 11306 & -004.5 & 19 & 17.1 & 15.9 & 1.2 & $\mathrm{Se}$ & & & \\
\hline 125 & 11306 & 004.9 & 5 & 19.0 & 18.0 & 1.1 & $?$ & & & LSB \\
\hline 126 & 11307 & -010.6 & 7 & 18.4 & 17.2 & 1.2 & $\mathrm{Sl}$ & & & \\
\hline 127 & 11309 & 004.4 & 8 & 18.4 & 16.5 & 1.9 & $\mathrm{E}$ & & & \\
\hline 128 & 11309 & 006.0 & 10 & 18.5 & 17.1 & 1.4 & $\mathrm{Se}$ & & & \\
\hline 129 & 11311 & -001.8 & 28 & 16.4 & 15.1 & 1.3 & $\mathrm{Se}$ & 53 & 13436 & COM? \\
\hline 130 & 11311 & -000.9 & 17 & 16.8 & 15.6 & 1.2 & $\mathrm{Se}$ & 52 & 13044 & \\
\hline 131 & 11311 & 004.1 & 23 & 16.1 & 15.1 & 1.0 & $\mathrm{Se}$ & 78 & & $\mathrm{~b}$ \\
\hline 132 & 11314 & -001.3 & 26 & 15.9 & 14.8 & 1.1 & $\mathrm{SB} e$ & 51 & & dist, sb, b-arm \\
\hline 133 & 11314 & 008.7 & 11 & 17.9 & 16.8 & 1.1 & $\mathrm{Se}$ & & & \\
\hline 134 & 11315 & -011.6 & 8 & 18.6 & 17.7 & 0.9 & $\mathrm{Se}$ & & & dist, b \\
\hline 135 & 11316 & $\begin{array}{ll}-0 & 09.0\end{array}$ & 13 & 16.0 & 14.6 & 1.4 & $\mathrm{E}$ & & & \\
\hline 136 & 11318 & -008.2 & 7 & 19.1 & 18.0 & 1.1 & $\mathrm{Se}$ & & & \\
\hline 137 & 11318 & -006.9 & 29 & 16.3 & 14.8 & 1.5 & $\mathrm{~S} l$ & 33 & & COM, r-comp \\
\hline 138 & 11318 & 000.8 & 7 & 19.4 & 18.4 & 1.0 & $\mathrm{Se}$ & & & $\mathrm{sb}$ \\
\hline 139 & 11320 & 006.7 & 12 & 17.9 & 16.9 & 0.9 & $\mathrm{Se}$ & & & $\mathrm{b}$ \\
\hline 140 & 11320 & 008.2 & 6 & 19.3 & 18.4 & 0.8 & $\mathrm{Sl} / \mathrm{I}$ & & & $\mathrm{b}$ \\
\hline 141 & 11322 & -011.2 & 9 & 17.1 & 16.3 & 0.8 & $\mathrm{Se} ?$ & 22,23 & & $\mathrm{DBL}, \mathrm{b}$ \\
\hline 142 & 11323 & -009.9 & 12 & 17.7 & 16.4 & 1.3 & $\mathrm{E}$ & & & \\
\hline 143 & 11323 & $\begin{array}{ll}0 & 02.2\end{array}$ & 10 & 18.0 & 16.7 & 1.3 & $\mathrm{E}$ & & & \\
\hline
\end{tabular}


Notes to TABLE 3

Columns:

(1) ID-number

(2), (3) Position of the galaxy center in 1950.0 equinox. The position accuracy is about 5 arcsec.

(4) Major axis diameter of the galaxies at $23 \mathrm{mag} \operatorname{arcsec}^{2}$ isophote in $I$ band.

(5), (6), (7) $V, I$, and $(V-I)$ mag. Tabulated $(V-I)$ colors do not always coincide with $V$ magnitude minus $I$ magnitude because of the round-off error.

(8) Eye inspected morphology; category is either of E, S0, early S [Sa - Sb] (Se), late S [Sc $\mathrm{Sd}](\mathrm{Sl})$, I. If we can see any bar structure, we add 'B'.

(9) The ID-number given in Dressler (1980).

(10) Measured heliocentric radial velocities.

(11) Some notes. We use the following abbreviations; LSB: low surface brightness; DBL: double system (the companion has the comparable size); COM: with a small companion (the number in parenthesis is the ID-number of the companion, if it is cataloged as another object in this list); comp: companion; dist: distortion; r: red; b: blue; sb: semi-blue; swb: somewhat blue. No. 39 is the only IRAS source and No. 81 is the only radio source; see text. 
TABLE 4. Distribution of the morphology.

\begin{tabular}{clrrccc}
\hline \hline Morphology $^{\mathrm{a}}$ & Number & Fraction $(\%)^{\mathrm{b}}$ & \multicolumn{2}{c}{ Blue galaxies } & \multicolumn{2}{c}{ Blue and semi-blue galaxies } \\
number & fraction $^{\mathrm{c}}$ & number & fraction $^{\mathrm{c}}$ \\
\hline $\mathrm{E}$ & 32.5 & 25 & 0 & 0 & 1 & 3 \\
$\mathrm{~S} 0$ & 12 & 9 & 0 & 0 & 2 & 17 \\
$\mathrm{Se}+\mathrm{SB} e$ & 74.5 & 58 & 15 & 20 & 29 & 39 \\
$\mathrm{~S} l+\mathrm{SB} l$ & 6.5 & 5 & 3 & 46 & 3 & 46 \\
$\mathrm{I}$ & 3.5 & 3 & 2 & 57 & 2 & 57 \\
$?$ & 14 & & 3 & 21 & 3 & 21 \\
\hline Total & 143 & 100 & 23 & 16 & 40 & 28 \\
\hline \hline
\end{tabular}

Notes to TABLE 4

${ }^{a}$ Barred and non-barred are combined together. Objects with uncertain assignments are also included. For objects assigned to two categories, we count a half of that in each category.

${ }^{\mathrm{b}}$ Fraction is that to the total number of objects with assigned morphologies, 129.

${ }^{c}$ Fraction is that to the total number in each morphological type. 